\title{
Risk factors for sporadic listeriosis in the Netherlands, 2008 to 2013
}

I H Friesema (ingrid.friesema@rivm.nl)², S Kuiling ${ }^{2}$, A van der Ende 3 , M E Heck², L Spanjaard³, W van Pelt ${ }^{1}$

1. Epidemiology and Surveillance of Infectious Diseases, Centre for Infectious Disease Control, National Institute for Public Health and the Environment (RIVM), Bilthoven

2. Infectious Diseases Research, Diagnostics and Screening, Centre for Infectious Disease Control, RIVM, Bilthoven

3. Department of Medical Microbiology, Netherlands Reference Laboratory for Bacterial Meningitis, Academic Medical Center, Amsterdam

Citation style for this article:

Friesema IH, Kuiling S, van der Ende A, Heck ME, Spanjaard L, van Pelt W. Risk factors for sporadic listeriosis in the Netherlands, 2008 to 2013 . Euro Surveill. 2015;20(31):pii=21199. Available online: http://www.eurosurveillance.org/ViewArticle. aspx?Articleld=21199

Although the disease burden of listeriosis on population level is low, on individual level the impact is high, largely due to severe illness and a high case fatality. Identification of risk factors supports and specifies public health actions needed for prevention. We performed a case-control study to determine host- and food-related risk factors for non-perinatal listeriosis in the Netherlands. Patients with non-perinatal listeriosis reported between July 2008 and December 2013 were compared with controls from a periodic control survey who completed a questionnaire in the same period. Higher age, male sex, underlying disease, especially cancer and kidney disease, and use of immunosuppressive medicine were strong risk factors for acquiring non-perinatal listeriosis. Analysis of the food consumption in the group of cases and controls with underlying diseases did not reveal any high-risk food products. Information and advice should continue to be given to persons at risk of severe listeriosis. Univariate analyses indicate that patients using gastric acid inhibitors are at risk. It is worth adding these patients to the group of susceptible persons.

\section{Introduction}

Listeria monocytogenes is widespread in nature, but human illness is almost always food-borne. Listeriosis is a rare but serious illness, mainly affecting elderly people, pregnant women and their unborn fetuses or newborn babies, and people with serious underlying disease [1-4]. The three most common clinical manifestations of listeriosis are febrile gastroenteritis, invasive illness (manifesting as bacteraemia, sepsis and/or meningitis) and maternal-fetal/neonatal listeriosis [2]. The disease burden of 14 food-borne pathogens in the Netherlands was assessed for 2009 [5]. The disease burden of listeriosis on population level was low (12th place; 96 disability adjusted life years (DALY) per year) but ranked second on individual level (1,220 DALY per 1,000 cases of illness), after toxoplasmosis and higher than for example salmonellosis and campylobacteriosis.

Identification of food products associated with listeriosis is difficult, because of the ubiquitous and psychrotrophic (cold-tolerant) nature of L. monocytogenes, the long and varying incubation period, and because severe illness is mainly restricted to vulnerable populations $[6,7]$. Outbreak investigations can provide information about risk products. In the case of listeriosis, outbreaks are often associated with errors during food production, such as contaminated slicing machines followed by opportunities for growth of the pathogen $[8,9]$. However, outbreak-related cases comprise only a small percentage of all listeriosis cases. In the Netherlands, no outbreak of listeriosis has been identified yet. Case-control studies of sporadic cases are useful for identifying risk factors, although it is more difficult to detect sources, as the cases do not necessarily share a single common source or food product [10].

Identification of risk factors for sporadic cases of listeriosis can help direct public health actions to prevent listeriosis, such as improved food safety messages for specific groups at risk. Here we performed a case-control study to determine host- and food-related risk factors for non-perinatal listeriosis in the Netherlands.

\section{Methods}

\section{Listeriosis surveillance}

In 2005, voluntary surveillance for human listeriosis was started in the Netherlands. Clinical microbiological laboratories were asked to report positive cultures of $L$. monocytogenes to the municipal health services. The health authorities contacted these patients with a questionnaire, and when possible interviewing them. If the patient had died or was too ill to be interviewed, spouses were interviewed when 


\section{FIGURE}

Incidence of non-perinatal listeriosis, by age group and sex, the Netherlands, July 2008-December $2013(\mathrm{n}=404)$

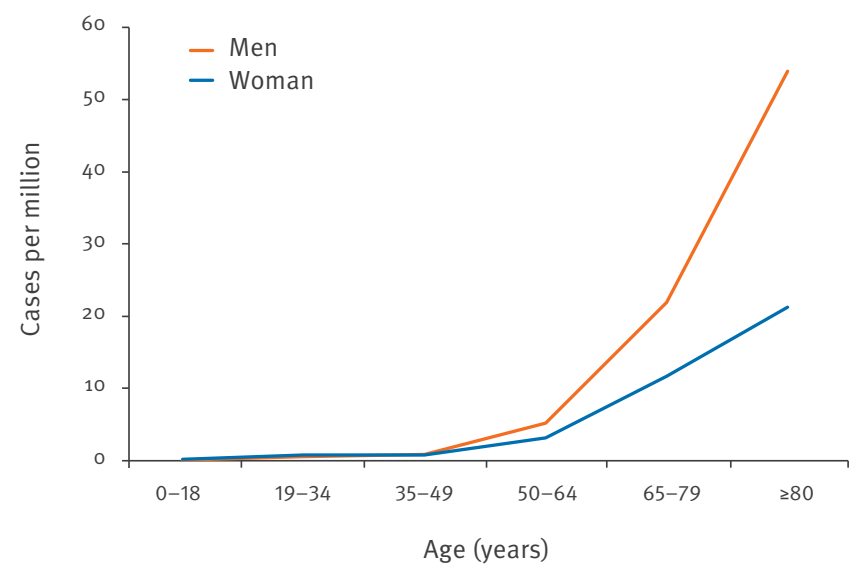

possible. The questionnaire contained items about underlying diseases and exposure to possible risk factors in the 30 days before the date of onset of the listeriosis. Listeriosis became a notifiable disease in the Netherlands in December 2008 and the procedure for voluntary surveillance was formalised. Unfortunately, the notification questionnaire with a focus on underlying disease and food consumption was shortened and now contains fewer food items.

Since 2005, clinical microbiological laboratories have been sending Listeria isolates from patients with invasive disease to the Netherlands Reference Laboratory for Bacterial Meningitis (NRLBM), which forwards the isolates to the National Institute for Public Health and the Environment (RIVM) for pulsed-field gel electrophoresis (PFGE) and serotyping. The laboratories can also send Listeria isolates from patients with other symptoms directly to the RIVM. This procedure did not change when voluntary surveillance became surveillance based on notifications in 2008.

\section{Control survey}

In July 2008 , a periodic control survey with a self-administered questionnaire was started in the Netherlands targeting the general population. The aim was to obtain data for identifying risk factors for several gastrointestinal, food-borne and respiratory infections. Three to four times a year, a random sample of the population is drawn and approached. The questionnaire comprises items about food consumption, contact with animals, travel and outdoor activities, health and underlying diseases and some demographic details. Only items phrased exactly the same as in the questionnaire used for the listeria case and administered in the same time period were included in the analyses. For a more detailed description of this control survey, see [11].

\section{Case-control study}

Cases and controls were included in the case-control study when they became ill or completed the questionnaire, respectively, between 1 July 2008 and 31 December 2013. This means that almost all listeriosis cases were reported via mandatory notification. Because the focus of the study was on non-perinatal listeriosis, pregnant women were excluded. Further exclusions applied to cases and controls were: (i) age 18 years or younger, as this group rarely develops listeriosis, (ii) travel abroad in the four weeks before illness or before completing the questionnaire, (iii) missing data on underlying disease or on use of immunosuppressants.

\section{Case-case comparison}

Also a case-case comparison was done, comparing infections with $L$. monocytogenes serotype $4 \mathrm{~b}$ with those with serotype $1 / 2 a$. The two groups of cases were analysed for differences in underlying disease and food products eaten.

\section{Data analysis}

Logistic analyses in the case-control study were done in two phases. Firstly, underlying diseases and use of medicines were analysed, adjusted for sex, age group (five categories), season (four categories) and level of urbanisation (five categories). All variables with $p$ values<0.1 were included in a multivariable model for further assessment, using backward selection. At each step, the least significant variable was removed from the model, until all variables in the model had reached significance $(p<0.05)$ and the model was significant. At every step, the estimated odds ratio (OR) for the remaining exposure(s) was checked for major changes compared with the previous step. If removal of a variable had led to a major change, the exposure would have been retained in the model. In the second phase of the logistic analyses, the food consumption of cases and controls was analysed only including persons with the underlying diseases and/or medicines used that remained in the final model of phase 1 . Food consumption consisted of 10 variables of meat products, eight fish and seafood products and five dairy products. Also, a variable was added about how often the respondent normally cleaned their refrigerator.

In the case-case comparison, underlying disease and food consumption were analysed together in one model, instead of the two phases that were applied in the case-control analyses. All analyses were done using SAS software, version 9.3 (SAS Institute).

\section{Results}

Between July 2008 and December 2013, 406 patients with non-perinatal listeriosis were reported, of whom 241 were men (59\%), 163 women $(40 \%)$ and two of unknown sex (0.5\%). Incidence of non-perinatal listeriosis increased with age, but the increase was more pronounced in men than in women (Figure). Incidence remained below 1 per million inhabitants up to the age of 50 years, and then increased to almost 54 (men) and 21 (women) per million inhabitants 80 years or older. Forty-one of 345 cases with known status died (12\%), 
TABLE 1

Characteristics of listeriosis patients $(\mathrm{n}=279)$ and control subjects $(\mathrm{n}=1,733)$, the Netherlands, July 2008-December 2013

\begin{tabular}{|c|c|c|}
\hline Characteristic & $\begin{array}{l}\text { Number of } \\
\text { cases (\%) }\end{array}$ & $\begin{array}{l}\text { Number of } \\
\text { controls (\%) }\end{array}$ \\
\hline $\mathrm{n}$ & 279 & 1,733 \\
\hline \multicolumn{3}{|l|}{ Sex } \\
\hline Male & $174(62)$ & $732(42)$ \\
\hline Female & $105(38)$ & $1,001(58)$ \\
\hline \multicolumn{3}{|l|}{ Age group } \\
\hline $19-34$ years & $10(4)$ & $154(9)$ \\
\hline $35-49$ years & $9(3)$ & $350(20)$ \\
\hline $50-64$ years & $59(21)$ & $598(35)$ \\
\hline $65-79$ years & $118(42)$ & $503(29)$ \\
\hline 80 or older & $83(30)$ & $128(7)$ \\
\hline \multicolumn{3}{|l|}{ Season } \\
\hline Winter (Dec-Feb) & $63(23)$ & $287(17)$ \\
\hline Spring (Mar-May) & $48(17)$ & $429(25)$ \\
\hline Summer (Jun-Aug) & $83(30)$ & $430(25)$ \\
\hline Autumn (Sep-Nov) & $85(30)$ & $587(34)$ \\
\hline \multicolumn{3}{|l|}{ Level of urbanisation } \\
\hline$\geq 2,500$ addresses $/ \mathrm{km}^{2}$ & $47(17)$ & $294(17)$ \\
\hline $1,500-2,499$ addresses $/ \mathrm{km}^{2}$ & $80(29)$ & 329 (19) \\
\hline $1,000-1,499$ addresses $/ \mathrm{km}^{2}$ & $59(21)$ & $367(21)$ \\
\hline 500-999 addresses $/ \mathrm{km}^{2}$ & $57(20)$ & $396(23)$ \\
\hline$<500$ addresses $/ \mathrm{km}^{2}$ & $36(13)$ & $347(20)$ \\
\hline
\end{tabular}

for 61 cases this was unknown. More men (28/205; $14 \%)$ than women (13/140; $9 \%$ ) died due to listeriosis (not statistically significant).

A total of 2,363 controls completed the questionnaire in the specified period. Three of the 406 patients and 287 controls were 18 years or younger, and were excluded from the case-control analyses. Furthermore, 19 cases and 267 controls had been abroad, and for a further 105 cases and 76 controls, no medical history was available. The case-control analysis therefore comprised 279 cases and 1,733 controls. Infection with L. monocytogenes serotype $4 \mathrm{~b}$ was most common $(n=98 ; 35 \%)$, followed by serotype $1 / 2 \mathrm{a}(n=67 ; 24 \%)$ and serotype $1 / 2 b(n=33 ; 12 \%)$; serotype $1 / 2 c(n=5 ; 2 \%)$ and $3 b$ $(n=1 ;<1 \%)$ were rarely seen, and for 75 patients $(27 \%)$ no serotype was available. Thirty-one of the 273 listeriosis cases included in the case-control analyses died (11\%), for six cases the outcome was unknown.

Characteristics of the patients and controls included in the case-control analyses are given in Table 1. Cases were more often male (62\%) than controls (42\%) and were older, with a median age of 72 years compared with 59 years.

Only a small number of cases ( $8 \%$ ) had no underlying disease, in contrast to the controls of whom $70 \%$ had no underlying disease (Table 2). Of the diseases included in the analyses, only diabetes, rheumatism and other underlying disease did not reach significance in the univariate analyses. Organ transplantation was not included in the multivariable analysis, as it showed high collinearity with immunosuppressants. In the final model, immunosuppressant use was the strongest risk factor (OR 53.7; 95\% Cl: 31.0-93.0), followed by cancer (OR 26.8; $95 \% \mathrm{Cl}: 14.4-49.8$ ) and chronic kidney disease (OR 21.8; 95\% Cl: 9.0-52.5).

A total of 246 cases ( $88 \%$ ) and 288 controls (17\%) had one of the underlying diseases from the final model or used immunosuppressants. Analysis of the food consumption in this group of cases and controls did not reveal any risk products. Eight of the 10 meat products, two of eight fish and seafood products and all five dairy products were eaten significantly less often by the cases than by the controls. The remaining eight products were eaten as often. No difference was seen in the frequency of cleaning the refrigerator.

Comparison of the cases with the two most commonly found serotypes, $4 \mathrm{~b}$ and $1 / 2 \mathrm{a}$, did not reveal any differences in underlying diseases or food consumption.

\section{Discussion}

In the present study, high age, male sex, underlying disease, especially cancer and kidney disease, and immunosuppressive medicine use were strong independent risk factors for acquiring non-perinatal listeriosis. These factors have been described before $[7,12,13]$. In a case-control study in Australia, usage of gastric acid inhibitors was identified as risk factor [14]. In the present study, gastric acid inhibitors were found a significant risk factor in the univariable analysis; in the multivariable model, it was no longer significant. This can be due to stronger effects of the comorbid factors that did remain in the final model. Bavishi and DuPont [15] concluded in their systematic review that the use of proton pump inhibitors can lead to bacterial colonisation and increased susceptibility to enteric bacterial infection. Although the evidence may still be weak for listeriosis, the biological plausibility of the effect of gastric acid inhibitors on the gastrointestinal system warrants caution in the use of these drugs, especially in already immunocompromised persons.

To exclude the impact of these host factors when examining high-risk food products, food consumption was analysed including only highly susceptible cases and controls (with underlying diseases or taking immunosuppressants). None of the food products could be labelled as risky food. This could be due to similar food advice given to both cases and controls; however, we had not asked about received food advice. Overall, identification of high-risk foods in a case-control study with sporadic cases can be difficult due to the ubiquity of the microorganism in the environment and fluctuating rates of contamination of food products, but also because some risky food products are frequently consumed in the control population, and 
TABLE 2

Underlying diseases and use of medicines in listeriosis patients $(\mathrm{n}=279)$ and control subjects $(\mathrm{n}=1,733)$, the Netherlands, July 2008-December 2013

\begin{tabular}{|c|c|c|c|c|}
\hline Characteristic & Number of cases (\%) & Number of controls (\%) & $\begin{array}{l}\text { Univariate OR } \\
(95 \% \mathrm{Cl})^{a}\end{array}$ & $\begin{array}{l}\text { Multivariable OR } \\
(95 \% \mathrm{Cl})^{\mathrm{a}}\end{array}$ \\
\hline \multicolumn{5}{|l|}{ Underlying disease } \\
\hline Diabetes & $50(18)$ & 158 (9) & $1.4(1.0-2.1)$ & \\
\hline Cardiovascular disease & $68(24)$ & $164(9)$ & $1.7(1.2-2.4)$ & $2.0(1.2-3.3)$ \\
\hline Immune disorder & $28(10)$ & $19(1)$ & $14.7(7.4-29.1)$ & $3.3(1.3-8.6)$ \\
\hline Cancer & $92(33)$ & $31(2)$ & $24.8(15.4-40.0)$ & $26.8(14.4-49.8)$ \\
\hline Chronic liver disease & $13(5)$ & $5(0.3)$ & $22.1(6.9-71.1)$ & $9.7(2.3-41.1)$ \\
\hline Lung disease & $40(14)$ & $93(5)$ & $2.1(1.3-3.2)$ & $-b$ \\
\hline Gastrointestinal disease & $32(11)$ & $64(4)$ & $4.2(2.5-7.0)$ & $2.1(1.0-4 \cdot 3)$ \\
\hline Chronic kidney disease & $38(14)$ & $13(1)$ & $18.2(8.8-37.7)$ & $21.8(9.0-52.5)$ \\
\hline Rheumatism & $26(9)$ & $78(5)$ & $1.5(0.9-2.6)$ & $-\mathrm{b}$ \\
\hline Organ transplant & $12(4)$ & $1(0.1)$ & $138.9(15.7-\infty)$ & ND \\
\hline Other underlying disease & $7(3)$ & $38(2)$ & $1.3(0.5-3.1)$ & $-\mathrm{b}$ \\
\hline No underlying disease & $22(8)$ & $1,217(70)$ & ND & ND \\
\hline \multicolumn{5}{|l|}{ Medicine use } \\
\hline Immunosuppressants & $162(58)$ & $41(2)$ & $80.3(49.8-129.7)$ & $53.7(31.0-93.0)$ \\
\hline $\begin{array}{l}\text { Gastric acid inhibitors; } \\
\text { 〈missing values〉 }\end{array}$ & $\begin{array}{l}94(43) \\
\quad\langle 60\rangle\end{array}$ & $\begin{array}{c}232(14) ; \\
\langle 57\rangle\end{array}$ & $3.8(2.7-5.3)$ & $-b$ \\
\hline
\end{tabular}

$\mathrm{Cl}$ : confidence interval; OR: odds ratio; ND: not determined

a OR adjusted for sex, age group, season and level of urbanisation.

${ }^{b}$ Not included in final multivariable model.

the varying incubation period $[7,10,13,14]$. Besides host and environmental components, virulence of the pathogen is another important risk factor [7]. Dose-response data are based on different animal and in vitro models and on epidemiological data from outbreaks which are scarce and have limitations. Another possibility for establishing food products as high-risk is by subtyping and comparing Listeria isolates found in humans and in food products [14]. Although finding similar strains in humans and in food does not prove causality, it could provide information about possible sources of infection and help us understand observed trends in human cases [16].

In our analyses, some food products generally recognised as risk foods for Listeria were significantly less often eaten by cases than by controls with similar host susceptibility. This probably indicates that persons with underlying diseases (cases and controls) have some knowledge about high-risk food and to some extent avoid eating such products. However, other often mentioned explanations include bias through differential recall of exposure between cases and controls or association of the food product with other unmeasured factors, such as the way and how long a product is stored at home. Furthermore, controls could possibly be less susceptible because underlying diseases are less frequent or less severe, or acquired (partial) immunity following frequent exposure to the high-risk food products [10]. Although it is assumed that immunity to L. monocytogenes in humans is long-lived, it has only been shown in mice [17-19].
Case-control studies in sporadic cases are commonly complex and resource-intensive [10]. In the present study, almost all cases in the case-control study were derived from the notification system as listeriosis has been a mandatory disease since December 2008. The controls originated from an ongoing control survey carried out since July 2008 [11]. This reduced substantially the extra work of seeking and contacting controls. A bias may have been introduced as most cases were personally interviewed by telephone while the controls received a self-administered questionnaire by post. Furthermore, the questionnaires to controls were sent on three defined dates per year, whereas cases occur throughout the year. The overall response rate in the control survey was $36 \%$ between July 2008 and December 2013. Analysis of the response in the period July 2008 to December 2012 showed a small underrepresentation of men, young people, people living in large cities and persons with both parents born outside the Netherlands [11]. Questioning persons can lead to exposure misclassification and recall bias [14]. Recalling food consumption more than four weeks in the past is difficult, especially when questioning spouses, and can make the respondents more likely to report usual food preferences than exact exposures.

Because of the ubiquity of the microorganism in the environment and the psychrotrophic nature of the bacterium, a wide variety of food products can become contaminated with L. monocytogenes and, when prepared without heating just before consumption, can infect a susceptible person [4]. Measures to minimise 
the chance of contamination at the time of consumption are use of high-quality ingredients, hygienic manufacturing practices, indication of appropriate shelf-life, correct refrigerated storage, education of food handlers and food service managers, and monitoring of food industry, catering and retail [8,20-22]. However, L. monocytogenes contamination cannot be entirely prevented. Thus, pregnant women, the elderly and persons with diseases and/or medications weakening the immune system should receive information about the presence of $L$. monocytogenes and advice about potential high-risk food products and how to handle food safely $[13,20]$. Due to the relatively high usage of gastric acid inhibitors and the biological plausibility of the effect on the gastrointestinal system, adding the group of users of gastric acid inhibitors to the high-risk groups should be considered.

\section{Conflict of interest}

None declared.

\section{Authors' contributions}

IF: collected and analysed the data from the epidemiological and molecular surveillance, coordinated the control survey and drafted the manuscript. AvdE / LS: contributed to the serotyping of the Listeria isolates received at the Netherlands Reference Laboratory for Bacterial Meningitis and sent the isolates to the National Institute for Public Health and the Environment, revised the manuscript and approved the final manuscript. SK / MH: contributed to the serotyping and PFGE of the Listeria isolates received at the National Institute for Public Health and the Environment, revised the manuscript and approved the final manuscript. WvP: supervised this study, contributed actively to the manuscript and approved the final manuscript.

\section{References}

1. Ramaswamy V, Cresence VM, Rejitha JS, Lekshmi MU, Dharsana KS, Prasad SP, et al. Listeria--review of epidemiology and pathogenesis. J Microbiol Immunol Infect. 2007;40(1):4-13. PMID:17332901

2. Drevets DA, Bronze MS. Listeria monocytogenes: epidemiology, human disease, and mechanisms of brain invasion. FEMS Immunol Med Microbiol. 2008;53(2):151 65. http://dx.doi.org/10.1111/j.1574-695X.2008.00404.X PMID:18462388

3. Awofisayo A, Amar C, Ruggles R, Elson R, Adak GK, Mook $P$, et al. Pregnancy-associated listeriosis in England and Wales. Epidemiol Infect. 2015;143(2):249-56. http://dx.doi. org/10.1017/So950268814000594 PMID:24650375

4. Allerberger F, Bagó Z, Huhulescu S, Pietzka A. Listeriosis: The dark side of refrigeration and ensiling. In: Sing A, editor. Zoonoses - Infections Affecting Humans and Animals Focus on Public Health Aspects. Heidelberg: Springer Verlag; 2015. p. 249-86.

5. Havelaar AH, Haagsma JA, Mangen MJ, Kemmeren JM, Verhoef LPB, Vijgen SMC, et al. Disease burden of foodborne pathogens in the Netherlands, 2009. Int J Food Microbiol. 2012;156(3):231-8. http://dx.doi.org/10.1016/j. ijfoodmicro.2012.03.029 PMID:22541392

6. Goulet V, King LA, Vaillant V, de Valk H. What is the incubation period for listeriosis? BMC Infect Dis. 2013;13(1):11. http:// dx.doi.org/10.1186/1471-2334-13-11 PMID:23305174

7. McLauchlin J, Mitchell RT, Smerdon WJ, Jewell K. Listeria monocytogenes and listeriosis: a review of hazard characterisation for use in microbiological risk assessment of foods. Int J Food Microbiol. 2004;92(1):15-33. http://dx.doi. org/10.1016/S0168-1605(03)00326-X PMID:15033265
8. Lianou A, Sofos JN. A review of the incidence and transmission of Listeria monocytogenes in ready-to-eat products in retail and food service environments. J Food Prot. 2007;70(9):217298. PMID:17900099

9. Todd ECD, Notermans S. Surveillance of listeriosis and its causative pathogen, Listeria monocytogenes. Food Contr. 2011;22(9):1484-90. http://dx.doi.org/10.1016/j. foodcont.2010.07.021

10. Fullerton KE, Scallan E, Kirk MD, Mahon BE, Angulo FJ, de Valk $\mathrm{H}$, et al. Case-control studies of sporadic enteric infections: a review and discussion of studies conducted internationally from 1990 to 2009. Foodborne Pathog Dis. 2012;9(4):281-92. http://dx.doi.org/10.1089/fpd.2011.1065 PMID:22443481

11. Friesema IHM, van Gageldonk-Lafeber $A B$, van Pelt W. Extension of traditional infectious disease surveillance with a repeated population survey. Eur J Public Health. 2015;25(1):1304. http://dx.doi.org/10.1093/eurpub/cku122 PMID:25085476

12. Gillespie IA, McLauchlin J, Grant KA, Little CL, Mithani V, Penman $C$, et al. Changing pattern of human listeriosis, England and Wales, 2001-2004. Emerg Infect Dis. 2006;12(9):1361-6. http://dx.doi.org/10.3201/eid1209.051657 PMID:17073084

13. Gillespie IA, Mook P, Little CL, Grant K, Adak GK. Listeria monocytogenes infection in the over-6os in England between 2005 and 2008: a retrospective case-control study utilizing market research panel data. Foodborne Pathog Dis. 2010;7(11):1373-9. http://dx.doi.org/10.1089/fpd.2010.0568 PMID:20586610

14. Dalton CB, Merritt TD, Unicomb LE, Kirk MD, Stafford RJ, Lalor K, et al. A national case-control study of risk factors for listeriosis in Australia. Epidemiol Infect. 2011;139(3):43745. http://dx.doi.org/10.1017/S0950268810000944 PMID:20429970

15. Bavishi C, Dupont HL. Systematic review: the use of proton pump inhibitors and increased susceptibility to enteric infection. Aliment Pharmacol Ther. 2011;34(11-12):126981. http://dx.doi.org/10.1111/j.1365-2036.2011.04874.x PMID:21999643

16. Lambertz ST, Ivarsson S, Lopez-Valladares G, Sidstedt M, Lindqvist R. Subtyping of Listeria monocytogenes isolates recovered from retail ready-to-eat foods, processing plants and listeriosis patients in Sweden 2010. Int J Food Microbiol. 2013;166(1):186-92. http://dx.doi.org/10.1016/j. ijfoodmicro.2013.06.008 PMID:23911759

17. Lavi O, Louzoun Y, Klement E. Listeriosis: a model for the fine balance between immunity and morbidity. Epidemiology. 2008;19(4):581-7. http://dx.doi.org/10.1097/ EDE.ob013e3181761f6f PMID:18496469

18. Lara-Tejero M, Pamer EG. T cell responses to Listeria monocytogenes. Curr Opin Microbiol. 2004;7(1):45-50. http:// dx.doi.org/10.1016/j.mib.2003.12.002 PMID:15036139

19. Pamer EG. Immune responses to Listeria monocytogenes. Nat Rev Immunol. 2004;4(10):812-23. http://dx.doi.org/10.1038/ nri1461 PMID:15459672

20. Luber P, Crerar S, Dufour C, Farber J, Datta AR, Todd ECD. Controlling Listeria monocytogenes in ready-to-eat foods: Working towards global scientific consensus and harmonization - Recommendations for improved prevention and control. Food Contr. 2011;22(9):1535-49. http://dx.doi. org/10.1016/j.foodcont.2011.01.008

21. Little CL, Pires SM, Gillespie IA, Grant K, Nichols GL. Attribution of human Listeria monocytogenes infections in England and Wales to ready-to-eat food sources placed on the market: adaptation of the Hald Salmonella source attribution model. Foodborne Pathog Dis. 2010;7(7):749-56. http://dx.doi. org/10.1089/fpd.2009.0439 PMID:20156087

22. Schuchat A, Deaver KA, Wenger JD, Plikaytis BD, Mascola L, Pinner RW, et al. Role of foods in sporadic listeriosis. I. Casecontrol study of dietary risk factors. JAMA. 1992;267(15):20415. http://dx.doi.org/10.1001/jama.1992.03480150047035 PMID:1552639 